\title{
Osteocondritis disecante. Primeros resultados en restos humanos de cazadores-recolectores del Holoceno en Patagonia austral
}

(跑) RMA

\author{
Osteochondritis dissecans. First results for Holocene hunter-gatherer \\ human remains from southern Patagonia \\ Rodrigo Zúñiga Thayer*, Jorge Suby**1, \\ Gustavo Flensborg ${ }^{\star * 2}$ y Leandro Luna***
}

Antropología Biológica

"Facultad de Filosofía y Letras, Universidad de Buenos Aires, Buenos Aires, Argentina. Grupo de Investigación en Bioarqueología. E-mail: rzunigat89@gmail.com

"INCUAPA-CONICET, Facultad de Ciencias Sociales, Universidad Nacional del Centro de la Provincia de Buenos Aires, Quequén, Argentina. Grupo de Investigación en Bioarqueología. E-mail: 1jasuby@gmail.com²gflensbo@soc.unicen.edu.ar ${ }^{* *} \mathrm{IMHICIHU}$-CONICET, Facultad de Filosofía y Letras, Universidad de Buenos Aires, Buenos Aires, Argentina. Grupo de Investigación en Bioarqueología. E-mail: lunaranda@gmail.com

\begin{abstract}
Resumen
La osteocondritis disecante (OCD) es una patología que afecta el cartílago articular y el hueso subcondral en las epífisis articulares de humanos y otros mamíferos. A pesar de que su etiología es multifactorial, la presencia de esta lesión ha sido frecuentemente atribuida a microtraumatismos reiterados por los efectos de la actividad física sobre las articulaciones afectadas. Este trabajo involucra un primer paso en el estudio de esta patología en restos humanos de Patagonia Austral, la cual no fue analizada en forma sistemática. El objetivo es analizar la variabilidad de la OCD en un conjunto de restos humanos de 26 individuos adultos pertenecientes a sociedades de cazadoresrecolectores de esta región durante el Holoceno medio-tardío (ca. 5200-70 años AP) y su posible relación con la edad, el sexo, la dieta, la cronología y el geográfica de procedencia. Se discute si su expresión está vinculada con los patrones de actividad física desarrollados por los grupos, en función de las prácticas económicas llevadas a cabo. La prevalencia general observada en esta muestra (42,3\%), mayor a la reportada para poblaciones actuales, sugiere que la actividad física habría desempeñado un rol importante en el desarrollo de la OCD en al menos parte de las personas afectadas.
\end{abstract}

Palabras clave: patologías articulares; paleopatología; actividad física; cazadores-recolectores; Patagonia.

\begin{abstract}
Osteochondritis dissecans (OCD) is a pathology that affects the articular cartilage and the subchondral bone in the articular epiphyses of humans and other mammals. Although its etiology is multifactorial, its presence has been frequently attributed to repetitive microtrauma because of physical activity on the affected joints. This paper is a first step in the study of this pathology in human remains from Patagonia Austral, as it has not been systematically studied. The objective is to analyze the variability of the OCD in a sample of 26 hunter-gatherer adult individuals that lived in this region during the Middle-Late Holocene (ca. 5200-70 years BP) and its possible relationship with age, sex, diet, chronology and region of origin. The association between its expression and the patterns of physical activity developed in accordance with the economic practices, is discussed. The overall prevalence observed in this sample (42,3\%), higher than that reported for current populations, suggests that physical activity would have played an important role in the development of OCD in at least part of the affected individuals.
\end{abstract}

Keywords: joint pathologies; paleopathology; physical activity; hunter-gatherers; Patagonia.

La osteocondritis disecante (OCD) es una condición caracterizada por la separación localizada de un fragmento del cartílago articular y el hueso subcondral, que afecta a las epífisis articulares de humanos y otros mamíferos (e.g. perros, gatos, cerdos, ratas, caballos, entre otros) (Edmonds y Heyworth 2014; Schenck y Goodnight 1996). Ha sido comúnmente estudiada en bioarqueología como un indicador asociado con la actividad física, aunque otros componentes etiológicos pueden estar involucrados (e.g. factores genéticos-hereditarios, variaciones anatómicas de las articulaciones, isquemia del cartílago durante el crecimiento o la vejez de un individuo; Bulgheroni et al. 2017; Bullough 2004; Ytrehus et al. 2007).

En Argentina, son escasas las investigaciones paleopatológicas que han considerado el análisis de la OCD en restos humanos, las cuales en general consistieron en relevamientos descriptivos de lesiones en individuos 
aislados, más que en estudios sistemáticos sobre conjuntos esqueletales de gran tamaño. Esta tendencia se repite en Patagonia Austral, donde a pesar de que la OCD fue diagnosticada en algunos individuos, no existen análisis particulares sobre esta patología. Por lo tanto, el objetivo de este trabajo es analizar la variabilidad de la OCD en un conjunto de restos humanos de individuos pertenecientes a sociedades de cazadores-recolectores de Patagonia Austral durante el Holoceno medio-tardío (ca. 5200-70el área de procedencia. En consecuencia, se discutirá si su expresión está vinculada con los patrones de actividad física desarrollados por los grupos humanos en función de las prácticas económicas llevadas a cabo. Al mismo tiempo se revisan los conocimientos clínicos actuales y los principales antecedentes bioarqueológicos de la OCD. Esta investigación forma parte de una tesis doctoral en curso (RZT), cuyo objetivo principal es evaluar y discutir el posible impacto de las estrategias de subsistencia desarrolladas durante el Holoceno tardío en Patagonia Austral sobre los patrones de actividad física y la variación en el uso del cuerpo.

\section{Terminología, patogénesis y epidemiología general de la OCD}

El desprendimiento de cuerpos intra-articulares fue inicialmente descripto por Paget (1870) y más extensamente explicado por Franz König en 1888, quien introdujo el término osteocondritis disecante (König 2013). Aunque aún sigue en uso, este término es considerado como incorrecto por algunos autores debido a que esta condición patológica no implica en sí misma el desarrollo de un proceso inflamatorio (Barrie 1987; Nagura 1960; Ytrehus et al. 2007). Se ha destacado que la OCD es una fase final de la osteocondrosis, que se inicia por una deficiencia en el aporte sanguíneo al hueso subcondral de las superficies articulares, seguida por un proceso necrosante (Ytrehus et al. 2007). La patogénesis de la osteocondrosis está compuesta por tres fases principales: 1) Osteocondrosis latens: uno o varios focos de necrosis, observables únicamente a través de corte histológico, producidos por isquemias durante el desarrollo del cartílago articular epifisial y que no afecta al tejido de hueso subcondral; 2) Osteocondrosis manifesta: las lesiones del cartílago no se mineralizan, impidiendo que el hueso subcondral se desarrolle con éxito y 3 ) Osteocondrosis dissecante: en las áreas de cartílago necrótico se desarrollan fisuras que pueden afectar al cartílago articular y al hueso subcondral subyacente, formando una solapa que permanece adherida o se desprende en la cavidad articular, dejando un cráter óseo en el hueso subcondral que refleja el tamaño original de la lesión (Thomas y Johannsen 2011; Ytrehus et al. 2007). Las dos últimas fases son las únicas visibles en el registro bioarqueológico y la distinción entre ellas no es posible en la mayoría de los casos, debido a la frecuente ausencia de cartílago articular. Por lo tanto, según algunos autores no resulta adecuado el diagnóstico específico de lesiones por osteocondritis disecante propiamente dicha (e.g. O'Connor 2008; Thomas y Johannsen 2011; Ytrehus et al. 2007).

Debido a esto, se ha sugerido que el término osteocondritis disecante, empleado comúnmente en paleopatología, debe ser remplazado por el de osteocondrosis (Appleby et al. 2015; Thomas y Johannsen 2011). Sin embargo, la osteocondrosis involucra también otros síndromes de naturaleza diferente a la OCD, que no incluyen el desprendimiento de cuerpos intrarticulares, sino otros procesos como por ejemplo el aumento de la cifosis vertebral en el Síndrome de Scheuermann (Lowe 1990) o la inflamación de la entesis posterior del calcáneo en el caso de la enfermedad de Sever (Hendrix 2005), entre varios otros. Debido a que el uso del término osteocondrosis para referirse exclusivamente a la osteocondritis disecante podría resultar al menos confuso en términos paleopatológicos, en este trabajo se continuará utilizando este último concepto, teniendo en cuenta que no es posible identificar sus estadios iniciales.

La etiología de la OCD es poco comprendida, debido a los múltiples factores que parecen estar involucrados en su desarrollo (Edmonds y Polowsky 2013; Ortner 2003; Vikatou et al. 2017). Las causas propuestas son la existencia de un componente genético hereditario que aumenta la predisposición a padecer esta patología (Padgett et al. 1995), posibles variaciones anatómicas en algunas articulaciones que provocan anormalidades en la transmisión de cargas mecánicas (Bulgheroni et al. 2017) y la isquemia del cartílago articular durante el crecimiento o la vejez de un individuo (Carlson et al. 1991; Ytrehus et al. 2004), aunque la mayor parte de los autores sostiene que la principal causa son los microtraumas repetitivos asociados con la actividad física (Edmonds y Polousky 2013; Ytrehus et al. 2007). Estos últimos explicarían la alta prevalencia de este tipo de lesiones en atletas de alto rendimiento (Aichroth 1971; Cahill 1995; Takahara et al. 1999). Por ejemplo, puede alcanzar entre un 2,1\% (Matsuura et al. 2014) y un 3,4\% en la tróclea del húmero en jugadores de béisbol (Kida et al. 2014). En cambio, en poblaciones no asociadas con la actividad física deportiva las frecuencias de OCD son menores, alcanzando por ejemplo entre un 0,01 y un 0,06\% en el caso de la rodilla (Lindén 1976; Petersen et al. 2006; Solomon et al. 2010). Es por este motivo que su presencia en restos bioarqueológicos ha sido comúnmente interpretada como evidencia de los efectos de la actividad física sobre las articulaciones afectadas (e.g. McWhirr et al. 1982; Rodríguez-Martín 2000; Vikatou 2012; Vikatou et al. 2017; Wells 1974).

A pesar de que puede desarrollarse en cualquier articulación sinovial del esqueleto, las más afectadas son la rodilla, el tobillo y el codo (en ese orden), mientras que es menos común en el hombro, las vértebras cervicales y la falange proximal del primer dedo del pie (Bullough 
2004; Edmonds y Heyworth 2014; Edmonds y Polousky 2013; Waldron 2009). A partir del análisis clínico de un conjunto de 452 individuos, Hefti et al. (1999) reportaron que en la rodilla, la cara lateral del cóndilo medial femoral es el sector más afectado (51\%), seguido por la cara central del mismo cóndilo (19\%), el cóndilo lateral femoral (17\%), la cara medial del cóndilo medial femoral (7\%) y la rótula (7\%). En cuanto a la OCD del tobillo, se observó a partir de un conjunto de 402 individuos que el 52,8\% corresponde a lesiones en la cara lateral del astrágalo, el $41,7 \%$ a la cara medial y el 5,5\% a la cara mediocentral (Schimmer et al. 2001; Berndt y Harty 1959). Por otro lado, entre 125 individuos con OCD en el codo, el 97,5\% de las lesiones afectó al cóndilo humeral (Kessler et al. 2013). Sin embargo, también se han reportado algunos casos de OCD en el olécranon, la cabeza radial y la tróclea (Eygendaal et al. 2017). En cuanto a la región del hombro, las porciones anatómicas más afectadas son la cabeza humeral y en menor medida la fosa glenoidea (e.g. Chu et al. 2009; Gogus y Ozturk 2008; Shanley y Mulligan 1990). En cualquiera de las articulaciones mencionadas, cuando no se realizan tratamientos médicos la OCD puede producir dolor y disminución en la capacidad física de la articulación afectada. Estudios de seguimiento de largo plazo mostraron además que puede provocar cambios degenerativos articulares (Uematsu et al. 2005).

Se ha propuesto que la OCD es más frecuente en individuos masculinos que en femeninos (Kocher et al. 2006; Lindén 1976; Schenck y Goodnight 1996). Este patrón se sugiere para todas las articulaciones, como lo demuestran numerosos estudios clínicos de rodilla (Desai et al. 1987; Jaberi 2002; Uematsu et al. 2005), del codo (Brownlow et al. 2006), del tobillo (Ming et al. 2004) y del hombro (Debeer y Brys 2005; Shanley y Mulligan 1990).

Por otra parte, no existe consenso acerca de los efectos de la edad en la prevalencia de la OCD, aunque esta patología parece influir en mayor medida a individuos que transitan las primeras etapas de la vida. La forma juvenil se presenta en niños y adolescentes entre 5 y 15 años de edad (Schenck y Goodnight 1996), la cual se asocia con el crecimiento y desarrollo óseo epifisial y con microtraumas repetitivos (e.g. Aichroth 1971; Anderson 2001; Edmonds y Polousky 2013; Polousky 2011; Takahara et al. 1999). La OCD juvenil puede no llegar a regenerarse después del desprendimiento de la porción necrótica, incluso durante la adultez (Cahill 1995; Edge y Porter 2011; Garrett 1991). Por el contrario, en adultos la OCD se inicia luego de finalizado el desarrollo epifisario y es poco frecuente en individuos mayores a los 50 años de edad (Garret 1991; Schenck y Goodnight 1996).

\section{Principales antecedentes bioarqueológicos}

En restos humanos esqueletizados, la OCD puede ser identificada macroscópicamente en las superficies articulares a través de la presencia de áreas focales osteonecróticas, dejando en evidencia un cráter o sequestrum óseo subcondral que puede alcanzar hasta 5 milímetros de profundidad y $2 \mathrm{~cm}$ de diámetro. Las porciones necróticas pueden encontrarse in situ o haber sido desprendidas, aunque el segundo caso es más frecuente (Aufderheide y Rodríguez-Martín 1998; Capasso et al. 1998; Ortner 2003; Waldron 2009).

A pesar de ser una patología frecuentemente estudiada en poblaciones actuales (Berndt y Harty 1959; Schenck y Goodnight 1996), la OCD ha sido objeto de un escaso número de trabajos específicos a partir de restos humanos arqueológicos (Tabla 1), por lo que posiblemente ha sido sub-diagnosticada en poblaciones del pasado. A su vez, son escasos los estudios sobre muestras que pertenecen a poblaciones cazadoras-recolectoras. Las principales investigaciones provienen de Europa a partir de conjuntos de restos humanos históricos y las prevalencias de OCD se encuentran entre el 0,8 y el 17,3\% (Bourbou 2003; Rodríguez-Martín 2000), afectando en mayor medida las articulaciones de la rodilla y del pie. En general, las frecuencias reportadas en conjuntos arqueológicos son mayores a las registradas en poblaciones actuales, aun respecto de aquellas relativas a las rodillas, los codos y los hombros en muestras conformadas por deportistas (Aichroth 1971; Cahill 1995; Takahara et al. 1999). Por lo tanto, en numerosas ocasiones las interpretaciones se refirieron a la actividad física como principal efecto causal.

En Argentina, las menciones a la OCD han sido particularmente escasas. Por ejemplo, Flensborg et al. (2011) describieron un individuo proveniente del sitio La Petrona procedente de la transición PampeanoPatagónica oriental afectado con OCD en el omóplato. Scabuzzo (2010) registró una lesión compatible con OCD bilateral en los omóplatos de un individuo recuperado en el sitio Paso Mayor Y1S2 del Sudoeste de la provincia de Buenos Aires. Además, Ratto et al. (2018) describieron OCD bilateral en los omóplatos en un individuo procedente del sitio Las Papas I (Tinogasta, Catamarca). En Patagonia Austral, este tipo de lesión articular ha sido mencionada en al menos tres ocasiones: en el omóplato de un individuo del sitio Santana 1 (Santiago et al. 2011), en el cúbito de un individuo recuperado en el sitio Rincón del Buque (Suby et al. 2009) y en un omóplato izquierdo del individuo proveniente del sitio Haberton Cementerio (Suby et al. 2011), todos ellos incluidos en este trabajo.

\section{Muestra y metodología}

La muestra está conformada por esqueletos adultos provenientes de Patagonia Austral (territorio sudamericano al Sur de la latitud $50^{\circ} \mathrm{S}$ ) con datos arqueológicos contextuales y preferentemente con información cronológica y paleodietaria. Se eligieron aquellos esqueletos que presentaron buena integridad y representación anatómica, es decir, los que contaron con más del $75 \%$ del esqueleto axial y apendicular, y sus 
articulaciones sinoviales en buen estado de preservación. No se incluyeron restos humanos asociados con cementerios de las misiones religiosas, debido a que los cambios en los modos de vida introducidos (e.g. Casali 2011; D’angelo del Campo et al. 2017; García Laborde et al. 2010) podrían generar sesgos en las interpretaciones. A su vez, teniendo en cuenta que, a diferencia de lo que ocurre en los adultos, la OCD en niños y adolescentes está más relacionada con el desarrollo somático que con la actividad física (Aichroth 1971; Anderson 2001; Edmonds y Polousky 2013; Shenk y Goodnight 1996; Takahara et al. 1999), en este trabajo se seleccionaron solo individuos adultos mayores de 18 años.

La estimación del sexo y de la edad de muerte fue realizada macroscópicamente en base a la inspección de rasgos morfológicos presentes en los elementos óseos considerados diagnósticos para este indicador (i.e. coxales, sacro y cráneo; Buikstra y Ubelaker 1994). El dimorfismo sexual de la pelvis también fue analizado de acuerdo con el método propuesto por Bruzek (2002). Para la estimación de la edad de muerte se analizaron los cambios morfológicos de la superficie auricular (Lovejoy et al. 1985; Osborne et al. 2004) y de la sínfisis púbica (Brooks y Suchey 1990; Suchey y Katz 1998; Todd 1921), y en ausencia de ellos, se observó el estado de obliteración de las suturas craneales (Meindl y Lovejoy 1989). Para el sacro se tuvo en cuenta el grado de fusión de las vértebras (Krogman e Isçan 1986).

La muestra quedó conformada por 26 individuos, 19 de ellos masculinos $(73,1 \%)$ y 7 femeninos $(26,9 \%)$. De ese total, 19 (73,1\%) son adultos jóvenes (20-35 años) y 7 (26,9\%) adultos medios (35-50 años) (Tabla 2). A su vez, 10 provienen de Santa Cruz-Magallanes (38,5\%), 9 del Norte de Tierra del Fuego $(34,6 \%)$ y 7 del Sur de Tierra del Fuego $(26,9 \%)$ (Figura 1). De los

Tabla 1. Estudios bioarqueológicos previos de osteocondritis disecante. Referencias: ND: no hay datos disponibles. Nota: los valores entre paréntesis en la columna de cronología son estimados por los autores de este trabajo

Table 1. Previous bioarchaeological studies of osteochondritis dissecans. References: ND: no data available. Note: the values in parentheses in the chronology column are estimated by the authors of this paper

\begin{tabular}{|c|c|c|c|c|c|}
\hline Referencias & Cronología & Procedencia & N & $\begin{array}{l}\text { Prevalencia } \\
\text { de OCD }\end{array}$ & Áreas afectadas \\
\hline Wells (1962) & $\begin{array}{l}\text { Periodo Anglosajón tardio } \\
\text { (ca. 1.051-884 años AP) }\end{array}$ & $\begin{array}{l}\text { Thorpe St. Catherine } \\
\text { (Inglaterra) }\end{array}$ & 1 & ND & Cóndilo medial de fémur: 1 \\
\hline Wells (1974) & $\begin{array}{l}\text { Edad del Bronce, periodos } \\
\text { Romano-británico y Anglosajón } \\
\text { (ca. 3.750-884 años AP) }\end{array}$ & $\begin{array}{l}\text { Colección arqueológica } \\
\text { del Museo de Castle } \\
\text { (Inglaterra) }\end{array}$ & 20 & ND & $\begin{array}{c}\text { Fémur (cóndilo): } 4 \\
1^{\circ} \text { Falange proximal: } 4 \\
\text { Tibia: } 3 \\
\text { Cúbito: } 2 \\
\text { Astrágalo: } 2 \\
\text { Atlas: } 1 \\
\text { Coxal (fosa glenoidea): } 1 \\
\text { Húmero: } 1 \\
\text { Navicular: } 1 \\
1^{\circ} \text { metatarso: } 1\end{array}$ \\
\hline McWhirr et al. (1982) & $\begin{array}{c}\text { Periodos Pre-romano, Romano } \\
\text { y Post-romano }\end{array}$ & $\begin{array}{l}\text { Cementerio de Bath } \\
\text { Gate (Inglaterra) }\end{array}$ & 362 & $39(10,8 \%)$ & $\begin{array}{c}\text { Rodilla: } 14 \\
1^{\circ} \text { falange proximal del Pie: } 7 \\
\text { Astrágalo: } 6 \\
\text { Hombro: } 6 \\
1^{\circ} \text { metatarso: } 6 \\
\text { Calcáneo: } 6 \\
\text { Tobillo: } 5 \\
\text { Vértebra: } 4 \\
\text { Cadera: } 2 \\
\text { Mandibula: } 1 \\
\text { Navicular del pie: } 1\end{array}$ \\
\hline Loveland et al. (1984) & $\begin{array}{l}\text { ca. } 1.550-1.700 \text { d.C. } \\
\text { (ca. } 400-250 \text { años AP)y } \\
\text { ca. } 1.350 \text { d.C. } \\
\text { (ca. } 600 \text { años AP) }\end{array}$ & $\begin{array}{l}\text { Sitios Kaufman-Williams } \\
\text { y Crow Creek (Estados } \\
\text { Unidos) }\end{array}$ & 3 & ND & $\begin{array}{l}\text { Fémur (cóndilo): } 1 \\
\text { Tarso: } 1 \\
\text { Metatarso: } 1\end{array}$ \\
\hline Stirland (1996) & $\begin{array}{l}\text { ca. } 1.245-1.468 \mathrm{~d} . C . \\
\text { (ca. } 705-482 \text { años AP) }\end{array}$ & $\begin{array}{l}\text { St. Margaret } \\
\text { Fyebridgegate } \\
\text { (Inglaterra) }\end{array}$ & 368 & $8(2,2 \%)$ & $\begin{array}{l}\text { Húmero (cabeza y cóndilo) y } \\
\text { Fémur (cabeza y cóndilos): } 8\end{array}$ \\
\hline Anderson (2000) & $\begin{array}{c}\text { Siglos XIII-XVI } \\
\text { (ca. 749-350 años AP) }\end{array}$ & $\begin{array}{l}\text { San Pietro Barisano } \\
\text { (Italia) }\end{array}$ & 1 & ND & Tarso: 1 \\
\hline Rodríguez-Martín (2000) & $\begin{array}{l}\text { Periodo prehispánico } \\
\text { (ca. } 2.550-500 \text { años AP) }\end{array}$ & $\begin{array}{c}68 \text { cuevas sepulcrales } \\
\text { Guanches de Tenerife } \\
\text { (España) }\end{array}$ & 1000 & $\begin{array}{c}173 \\
(17,3 \%)\end{array}$ & $\begin{array}{c}\text { Rodilla: } 6,02 \% \\
\text { Pie: } 4,56 \% \\
\text { Tobillo: } 3,05 \% \\
\text { Codo: } 2,35 \% \\
\text { Mano: 1,19\% } \\
\text { Hombro: 0,73\% } \\
\text { Muñeca: } 0,53 \% \\
\text { Cadera: } 0,31 \%\end{array}$ \\
\hline Anderson (2001) & $\begin{array}{c}\text { Siglos XIII-XVI } \\
\text { (ca. 749-350 años AP) }\end{array}$ & $\begin{array}{l}\text { St. Faith's Lane, } \\
\text { Norwich, (Inglaterra) }\end{array}$ & 1 & ND & Segunda cuña: 1 \\
\hline Silva (2001) & $\begin{array}{l}\text { Neolítico y Calcolítico } \\
\text { (ca. 6.750-4.250 años AP) }\end{array}$ & $\begin{array}{l}\text { Sitios Dólmen de } \\
\text { Ansião, Serra da Roupa, } \\
\text { Tholos de Paimogo I, } \\
\text { Cova da Moura, Cabeço } \\
\text { da Arruda Il e Hipogeu } \\
\text { de São Paulo (Portugal) }\end{array}$ & 908 & $8(0,9 \%)$ & $\begin{array}{l}\text { Cúbito (cavidad sigmoidea): } 3 \\
\text { Cóndilo femoral: } 1 \\
\text { Cabeza femoral: } 1 \\
\text { Tibia (cavidad glenoidea): } 1 \\
\text { Tibia (epífisis distal): } 1 \\
\text { Coxal (fosa glenoidea): } 1\end{array}$ \\
\hline Bourbou (2003) & $\begin{array}{l}\text { ca. } 500-700 \text { d.C. } \\
\text { (ca. } 1.450-1.250 \text { años AP) }\end{array}$ & $\begin{array}{c}\text { Eleuterna y Mesene } \\
\text { (Grecia) }\end{array}$ & 225 & $2(0,8 \%)$ & $\begin{array}{c}\text { Omóplato (fosa glenoidea): } 1 \\
\text { Tibia (cóndilo): } 1\end{array}$ \\
\hline Ortner (2003) & $\begin{array}{c}\text { Edad Media } \\
\text { (ca. 1.474-497 años AP) }\end{array}$ & $\begin{array}{l}\text { Iglesia St. George } \\
\text { (Inglaterra) }\end{array}$ & 1 & ND & Fémur (cóndilo medial): 1 \\
\hline $\begin{array}{l}\text { Kothari et al. (2009) } \\
\text { Ponce (2010) }\end{array}$ & ca. 4.000 años AP & El Morro de Arica (Chile) & 1 & ND & Cóndilo medial de fémur (bilateral): 1 \\
\hline Suby et al. (2009) & $\begin{array}{c}\text { Holoceno tardio } \\
\text { (ca.1.750-70 años AP) }\end{array}$ & $\begin{array}{c}\text { Rincón de Buque, } \\
\text { Cañadón Misionero, } \\
\text { Puerto Santa Cruz, Bajo } \\
\text { Nuevo y Punta Entrada } \\
\text { (Argentina) }\end{array}$ & 8 & ND & Cúbito (cavidad sigmoidea): 1 \\
\hline Scabuzzo (2010) & $\begin{array}{l}\text { Holoceno tardio } \\
\text { ca. } 700 \text { años AP }\end{array}$ & $\begin{array}{c}\text { Paso Mayor Y1S2 } \\
\text { (Argentina) }\end{array}$ & $\begin{array}{c}7 \\
\text { (NMI) }\end{array}$ & ND & Omóplato (fosa glenoidea): 2 \\
\hline Šlaus et al. (2010) & $\begin{array}{c}\text { Siglo VIII d.C } \\
\text { (ca. } 1.249-1.150 \text { años AP) }\end{array}$ & $\begin{array}{l}\text { Cementerio de Gluvine } \\
\text { kuće (Croacia) }\end{array}$ & 1 & ND & Cóndilo medial de fémur (bilateral): 1 \\
\hline Flensborg et al. (2011) & $\begin{array}{c}\text { Holoceno tardio } \\
\text { (ca. } 800-250 \text { años AP) }\end{array}$ & La Petrona (Argentina) & 8 & ND & Omóplato (fosa glenoidea): 1 \\
\hline Santiago et al. (2011) & $\begin{array}{l}\text { Holoceno medio-tardio } \\
\text { (ca. 5.200-250 años AP) }\end{array}$ & $\begin{array}{l}\text { Puerto Pescador 1, } \\
\text { Santana 1, Chorrillos (1 } \\
\text { y 2), La Arcillosa 2, Pozo } \\
\text { Tierra del Fuego 1, } \\
\text { Cantera Rhasa y Margen } \\
\text { Sur (Argentina) }\end{array}$ & 1 & ND & Omóplato (fosa glenoidea): 1 \\
\hline Suby et al. (2011) & ca. $1.536-70$ años AP & $\begin{array}{c}\text { Acatushun, Harberton } \\
\text { Cementerio, Paiashauaia } \\
\text { 1, Imiwaia (1 y 2), } \\
\text { Mischiuen 3, } \\
\text { Shamakush (Entierro } \\
\text { 1,2,3,4 y 6) (Argentina) }\end{array}$ & 1 & ND & Omóplato (fosa glenoidea): 1 \\
\hline $\begin{array}{c}\text { Vikatou (2012) } \\
\text { Vikatou et al. (2017) }\end{array}$ & $\begin{array}{l}\text { ca. } 1.618-1.866 \mathrm{~d} . \mathrm{C} . \\
\text { (ca. 332-84 años AP) }\end{array}$ & $\begin{array}{c}\text { Cementerio de } \\
\text { Middenbeemster (Paises } \\
\text { Bajos) }\end{array}$ & 93 & $14(15,1 \%)$ & $\begin{array}{c}1^{\circ} \text { falange proximal del pie: } 9 \\
1^{\circ} \text { metatarso: } 6 \\
\text { Astrágalo: } 3 \\
\text { Navicular pie: } 2\end{array}$ \\
\hline Darton et al. (2017) & $\begin{array}{c}\text { Siglo VII } \\
\text { (ca. 1.349-1.250 años AP) }\end{array}$ & Poses (Francia) & 1 & ND & Fémur (cóndilo lateral): 1 \\
\hline Ratto et al. (2018) & ca. $1.243 \mathrm{AP}$ & $\begin{array}{l}\text { Las Papas I (Fiambalá, } \\
\text { Catamarca, Argentina) }\end{array}$ & 1 & ND & Omóplato (fosa glenoidea): 1 \\
\hline
\end{tabular}


Tabla 2. Restos humanos analizados. Referencias: M: masculino; F: Femenino; AM: Adulto medio; T: Terrestre; Mt: Mixta; M: Marítima; ND: no hay datos disponibles Las cronologías expresadas con números se presentan en años antes del presente a partir de fechados radiocarbónicos no calibrados

Table 2. Human remains analyzed. References: M: male; F: Female; AM: Middle adult; T: Terrestrial; Mt: Mixed; M: Maritime; ND: no data available The chronologies expressed with numbers are shown in years before the present from uncalibrated radiocarbon dates

\begin{tabular}{|c|c|c|c|c|c|c|}
\hline Región & Sitio & Cronología & Sexo & Edad (años) & Dieta & Referencia \\
\hline \multirow{10}{*}{$\begin{array}{c}\text { Santa Cruz- } \\
\text { Magallanes } \\
\mathrm{N}=10\end{array}$} & Cañadón Misionero & $70 \pm 30$ & $\mathrm{M}$ & $35-45$ & $\mathrm{~T}$ & Suby et al. (2009) \\
\hline & Rincón del Buque & $830 \pm 42$ & M & $35-40$ & $\mathrm{~T}$ & Suby et al. (2009) \\
\hline & Cabo Vírgenes 17 & $900 \pm 40$ & M & 20-34 & $\mathrm{T}$ & L'Heureux et al. (2003) \\
\hline & Cerro Johnny & $390 \pm 60$ & M & $30-40$ & $\mathrm{~T}$ & Borrero y Barberena (2006) \\
\hline & Juni Aike & Contacto & M & $30-40$ & $\mathrm{~T}$ & Borrero y Barberena (2006) \\
\hline & Orejas de Burro 1 & $3565 \pm 45$ & M & $20-25$ & $\mathrm{~T}$ & L'Heureux y Barberena (2008) \\
\hline & Orejas de Burro 2 & $3565 \pm 45$ & M & $45-50$ & $\mathrm{~T}$ & L'Heureux y Barberena (2008) \\
\hline & Puerto Natales 3 & Siglo XIX & $\mathrm{F}$ & $20-25$ & ND & Prieto (1993-1994) \\
\hline & Punta Daniel & $1118 \pm 43$ & M & $30-40$ & Mt & Suby (2014) \\
\hline & Posesión Olimpia 2 & Siglo XIX & M & $30-40$ & $\mathrm{~T}$ & Suby (2014) \\
\hline \multirow{9}{*}{$\begin{array}{c}\text { Norte de } \\
\text { Tierra del } \\
\text { Fuego } \\
\mathrm{N}=9\end{array}$} & Lengua de Vaca & $251 \pm 41$ & $\mathrm{~F}$ & $30-40$ & Mt & Suby (2014) \\
\hline & Las Mandibulas & Siglo XIX & M & $20-24$ & $T$ & Guichón et al. (2000) \\
\hline & Bahía Gente Grande & Contacto & M & $30-40$ & $\mathrm{~T}$ & Suby (2014) \\
\hline & Bahía Felipe 1 & $1608 \pm 45$ & M & $35-50$ & Mt & Suby (2014) \\
\hline & Bahía Felipe 2 & $1608 \pm 45$ & $\mathrm{~F}$ & $30-40$ & Mt & Suby (2014) \\
\hline & La Arcillosa 2 & $5208 \pm 58$ & $\mathrm{~F}$ & $20-24$ & $\mathrm{~T}$ & Santiago et al. (2011) \\
\hline & Myren 1 & $640 \pm 20$ & M & $18-23$ & Mt & Suby (2014) \\
\hline & Puesto Pescador & $335 \pm 35$ & M & $21-25$ & $\mathrm{~T}$ & Suby et al. (2009) \\
\hline & Santana 1 & $269 \pm 46$ & M & $35-50$ & $\mathrm{~T}$ & Santiago et al. (2011) \\
\hline \multirow{7}{*}{$\begin{array}{c}\text { Sur de Tierra } \\
\text { del Fuego } \\
\quad \mathrm{N}=7\end{array}$} & Acatushún & Contacto & $\mathrm{F}$ & $30-40$ & M & Piana et al. (2006) \\
\hline & Harberton Cementerio & Contacto & M & 25-35 & M & Suby et al. (2011) \\
\hline & Paiashauaia 1 & $1504 \pm 46$ & $\mathrm{~F}$ & $35-45$ & $\mathrm{M}$ & Suby et al. (2011) \\
\hline & Aleph 3, Bahía Tetys & ND & M & 30-39 & M & Tessone et al. (2011) \\
\hline & Caleta Falsa 8-4 & ND & M & $43-49$ & ND & Guichón y Suby (2011) \\
\hline & Caleta Falsa 7-2 & $820 \pm 40$ & $\mathrm{~F}$ & $30-39$ & M & Guichón y Suby (2011) \\
\hline & Caleta Falsa 8-1 & ND & $M$ & $18-23$ & ND & Guichón y Suby (2011) \\
\hline
\end{tabular}

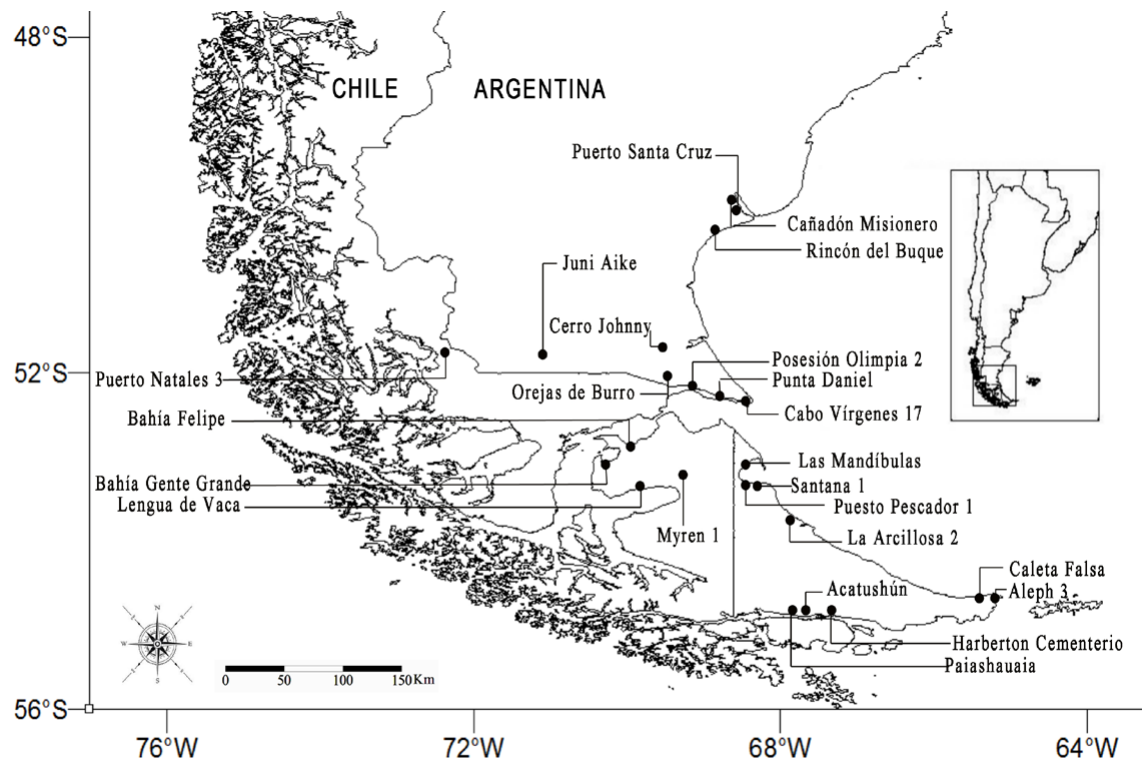

Figura 1. Localización de los sitios arqueológicos de los cuales provienen los restos humanos analizados en este trabajo

Figure 1. Location of the archaeological sites from which the human remains analyzed in this work come from

esqueletos analizados, solo 23 cuentan con información paleodietaria a partir de isótopos estables. De ese total, $13(56,6 \%)$ corresponden a una dieta considerada terrestre, $5(21,7 \%)$ a una dieta mixta y los 5 restantes $(21,7 \%)$ a una dieta con predominancia de consumo de recursos marítimos según estudios previos realizados por otros autores (Tabla 2). También 23 esqueletos tienen información cronológica, ya sea a partir de datos radiocarbónicos o contextuales. De esos individuos, 11 $(47,8 \%)$ corresponden al periodo pre-contacto, mientras los restantes 12 (52,2\%) pertenecen al periodo de contacto.
Se consideraron como OCD a las depresiones en forma de cráter, circular y ovalada, identificadas en los extremos distales o proximales de todas las articulaciones sinoviales (Aufderheide y Rodríguez-Martín 1998; Capasso et al. 1998; Ortner 2003; Waldron 2009). Todas las depresiones fueron medidas mediante el uso de un calibre digital. También se exploró la variabilidad en la prevalencia de OCD respecto del sexo y de la edad de muerte de los individuos y se analizó la posible relación entre la presencia de OCD y el tipo de economía, inferida a partir de los análisis de isótopos estables del carbono y nitrógeno realizados por otros investigadores (Tabla 2). Los esqueletos fueron agrupados siguiendo tres patrones dietarios basados en estudios isotópicos publicados previamente: terrestre, marítimo y mixto, este último interpretado como aquel en el cual los recursos marítimos representan entre el $20 \%$ y el $70 \%$ de la dieta consumida (Barberena 2002; Borrero y Barberena 2006; Borrero et al. 2009). Asimismo, se analizó la relación de la frecuencia de OCD con la cronología de los restos humanos estudiados, clasificados en pre-contacto (antes de los 400 años AP) y durante el contacto (después de los 400 años AP). Por último, se evaluó la posible diferencia de OCD de acuerdo con las diferentes de procedencia de los entierros, clasificadas en Santa Cruz/Magallanes, Norte de Tierra del Fuego y Sur de Tierra del Fuego, cuya subdivisión se basa en la variabilidad cultural y biológica que caracteriza a la región de estudio (e.g. GonzálezJosé et al. 2001; Guichón 1994; Saletta 2015). Todas las diferencias fueron estadísticamente testeadas mediante el Test de Fisher de dos colas, empleando el programa Statistica 7.0.

\section{Resultados}

Se registraron lesiones atribuibles a OCD en 11 de los 26 individuos analizados en este trabajo (42,3\%). Todas se presentaron en la superficie subcondral interarticular como forámenes de aproximadamente $3 \mathrm{~mm}$ de diámetro y entre 2 y $3 \mathrm{~mm}$ de profundidad, de bordes suaves y redondeados, lo que sugiere su estado remodelado, sin el fragmento óseo interno (Figura 2). Fueron identificadas 
en total 15 lesiones: 10 (66,7\%) en el hombro, afectando la fosa glenoidea del omóplato (6 derechos y 4 izquierdos, de los cuales son bilaterales en 2 individuos), 3 (20\%) en huesos del pie ( 2 de ellas bilaterales), registradas en la falange proximal del primer dedo, $1(6,7 \%)$ en el codo, sobre la epífisis proximal del cúbito derecho y 1 $(6,7 \%)$ en la faceta articular derecha de un axis (Tabla 3; Figuras 2 y 3). No se observaron lesiones en los otros elementos que conforman cada articulación afectada ni cambios degenerativos en las articulaciones con OCD (i.e. porosidad, formación de hueso nuevo marginal o en la superficie articular y alteraciones del contorno articular; Waldron 2009).

Las OCD en la cavidad glenoidea del omóplato solo afectaron a individuos masculinos, al igual que la lesión hallada en el cúbito del esqueleto recuperado en el sitio Rincón del Buque. Además, 8 de las 10 OCD halladas en la fosa glenoidea del omóplato fueron registradas en individuos de Santa Cruz/Magallanes y el Norte de Tierra del Fuego, mientras que las dos restantes se observaron en esqueletos procedentes del Sur de Tierra del Fuego (Tabla 3). La única OCD registrada en un individuo femenino involucró la articulación proximal de la falange proximal del primer dedo del pie derecho.

La OCD afectó en mayor medida a los hombres (aunque sin diferencias estadísticamente significativas respecto a las mujeres; $p=0,39)$, dado que 10 de los 19 individuos masculinos $(52,6 \%)$ y 1 de los 7 femeninos $(14,3 \%)$ presentaron al menos una lesión. Con respecto a la edad de muerte, los resultados tampoco resultaron en diferencias significativas. Sin embargo, se observó que 7 de los 19 adultos jóvenes (36,8\%) y 4 de los 7 adultos medios $(57,1 \%)$ presentaron lesiones (Tabla $4 ; p=0,69)$. Desde el punto de vista cronológico, 5 de los 11 individuos del periodo pre-contacto $(45,4 \%)$ y 5 de 12 individuos del periodo de contacto $(41,7 \%)$ presentaron OCD $(p=1)$, aunque nuevamente estas diferencias no resultaron significativas. El individuo recuperado en el sitio Caleta Falsa 8-1, en el cual se registró la presencia de OCD bilateral en las fosas glenoideas de ambos omoplatos, no cuenta con información cronológica, por lo que no pudo ser incluido en esta sección del análisis. Finalmente, en base a la dieta se registraron alteraciones articulares en 8 de los 13 individuos asociados a componentes terrestres (61,5\%), 2 de los 5 vinculados con una dieta mixta (40\%) y solo 1 de 5 con dieta marítima (20\%) (Tabla 4). A pesar de estas diferencias porcentuales, ninguna resultó significativamente diferente entre sí (terrestre vs. mixta $p=1$; terrestre vs. marítima $p=0,3$; mixta vs. marítima $p=1$ ).

Para el área de Santa Cruz/Magallanes, 6 de 10 individuos (60\%) presentaron OCD (Tabla 4). Entre los individuos de esta, se observaron lesiones en 6 de 9 individuos de sexo masculino $(66,7 \%)$, mientras que no se identificaron entre los femeninos. Cuatro de 7 individuos adultos jóvenes $(57,1 \%)$ y 2 de 3 individuos adultos medios $(66,7 \%)$ presentaron lesiones de OCD, así como 4 de 5 individuos del periodo pre-contacto (80\%) y 2 de 5 individuos del periodo durante el contacto (40\%). Considerando la información paleodietaria, 8 de 13 individuos asociados con una dieta terrestre $(61,5 \%)$ mostraron lesiones, mientras solo 2 de los 5 (40\%) con dietas mixtas y 1 de los 5 (20\%) con dietas marítimas presentaron OCD en al menos una articulación.

En el caso del área correspondiente al Norte de Tierra del Fuego, 4 de los 9 (44,4\%) individuos analizados tienen este tipo de lesiones (Tabla 4). Estas fueron observadas en 3 de los 6 individuos masculinos (50\%) y en solo 1 de los 3 femeninos (33,3\%). Dos de los 7 adultos jóvenes

Tabla 3. Individuos analizados y ubicación de las lesiones identificadas. Referencia: SL: sin lesiones

Table 3. Individuals analyzed and location of the identified lesions. Reference: SL: no lesions

\begin{tabular}{|c|c|c|c|c|}
\hline Región & Sitio & $O C D$ & Nro. De Lesiones & Localización \\
\hline \multirow{10}{*}{$\begin{array}{c}\text { Santa Cruz- } \\
\text { Magallanes } \\
\mathrm{N}=10\end{array}$} & Cañadón Misionero & No & 0 & SL \\
\hline & Rincón del Buque & Sí & 1 & Epífisis proximal del cúbito derecho \\
\hline & Cabo Vírgenes 17 & Sí & 1 & Fosa glenoidea del omóplato derecho \\
\hline & Cerro Johnny & No & 0 & SL \\
\hline & Juni Aike & Si & 3 & $\begin{array}{l}\text { Falange proximal del primer dedo del pie (bilateral) y } \\
\text { faceta articular derecha del axis }\end{array}$ \\
\hline & Orejas de Burro 1 & Sí & 1 & Fosa glenoidea del omóplato izquierdo \\
\hline & Orejas de Burro 2 & Sí & 1 & Fosa glenoidea del omóplato izquierdo \\
\hline & Puerto Natales 3 & No & 0 & SL \\
\hline & Punta Daniel & No & 0 & SL \\
\hline & Posesión Olimpia 2 & Sí & 1 & Fosa glenoidea del omóplato derecho \\
\hline \multirow{9}{*}{$\begin{array}{c}\text { Norte de } \\
\text { Tierra del } \\
\text { Fuego } \\
\mathrm{N}=9\end{array}$} & Lengua de Vaca & Si & 1 & Primera falange del primer dedo del pie derecho \\
\hline & Las Mandibulas & Sí & 1 & Fosa glenoidea del omóplato derecho \\
\hline & Bahía Gente Grande & No & 0 & SL \\
\hline & Bahía Felipe 1 & Si & 2 & Fosa glenoidea del omóplato (bilateral) \\
\hline & Bahía Felipe 2 & No & 0 & SL \\
\hline & La Arcillosa 2 & No & 0 & SL \\
\hline & Myren 1 & No & 0 & SL \\
\hline & Puesto Pescador & No & 0 & SL \\
\hline & Santana & Si & 1 & Fosa glenoidea del omóplato derecho \\
\hline \multirow{7}{*}{$\begin{array}{c}\text { Sur de } \\
\text { Tierra del } \\
\text { Fuego } \\
\mathrm{N}=7\end{array}$} & Acatushún & No & 0 & $S L$ \\
\hline & Harberton Cementerio & No & 0 & SL \\
\hline & Paiashauaia & No & 0 & SL \\
\hline & Bahia Tetys & No & 0 & SL \\
\hline & Caleta Falsa 8-4 & No & 0 & SL \\
\hline & Caleta Falsa 7-2 & No & 0 & SL \\
\hline & Caleta falsa 8-1 & Sí & 2 & Fosa glenoidea del omóplato (bilateral) \\
\hline
\end{tabular}

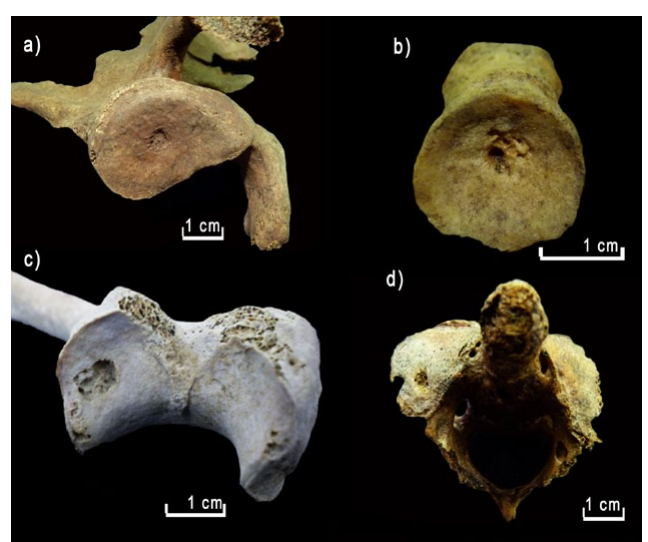

Figura 2. Elementos afectados por OCD en los restos humanos analizados. a) omóplato; b) primera falange proximal del primer dedo del pie; c) cúbito; d) axis

Figure 2. Elements affected by OCD in the human remains analyzed. a) scapula; b) first proximal phalanx of the first toe; c) ulna; d) axis 


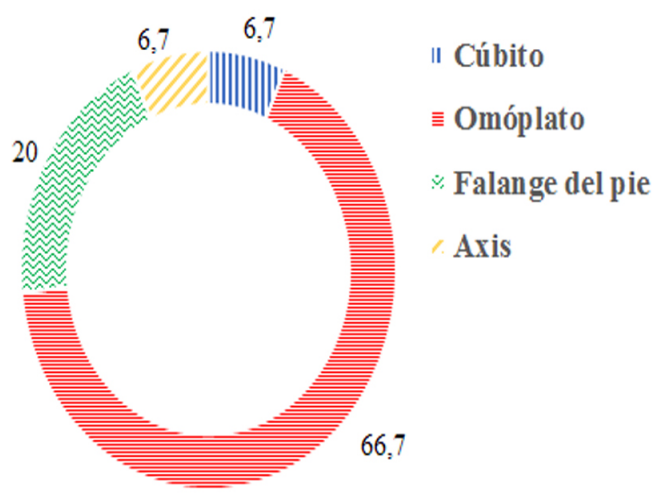

Figura 3. Porcentaje de afectación articular por OCD en los restos humanos analizados

Figure 3. Percentage of joint involvement by OCD in the human remains analyzed

$(28,6 \%)$ y los 2 adultos medios recuperados en esta región presentaron lesiones de OCD. En relación con la información cronológica disponible, 1 de los 4 individuos del periodo pre-contacto $(25 \%)$ y 3 de los 5 del periodo durante el contacto (60\%) evidencian este tipo de lesión. De acuerdo con la información paleodietaria, se observaron lesiones en 2 de los 5 individuos asociados con dieta terrestre (40\%) y en 2 de los 4 con dieta mixta (50\%). Por último, en el área del Sur de Tierra del Fuego, solo 1 de los 7 individuos (14,3\%) presentó lesiones de OCD (Tabla 4). Este masculino adulto joven tiene una dieta marítima y no se dispone de información cronológica.

\section{Discusión}

La presencia de OCD en restos humanos arqueológicos ha sido comúnmente interpretada como evidencia de la realización de actividades físicas intensas (e.g. McWhirr et al. 1982; Ortner 2003; Ponce 2010; RodríguezMartín 2000; Vikatou 2012; Vikatou et al. 2017; Wells 1974). Aunque esta causa puede estar involucrada, la complejidad de la etiología de la OCD obliga a considerar otras alternativas. Al mismo tiempo, ajustes metodológicos y terminológicos resultan necesarios al momento de analizar este tipo de lesiones, debido a las diferentes etapas de su patogénesis (Appleby et al. 2015). Respecto de las metodologías, las propuestas vigentes actualmente no permiten evaluar la extensión y severidad de las lesiones; en todos los casos proporcionan solo análisis descriptivos, como los mencionados por Aufderheide y Rodríguez-Martín (1998) y Ortner (2003). En relación con la terminología, aunque algunos han propuesto el uso del concepto de osteocondrosis, actualmente se reconoce que puede generar confusiones con otras patologías de naturaleza diferente y que no involucran las regiones articulares sinoviales. Por este motivo, el término osteocondritis disecante resulta más adecuado. De todos modos, es necesario incorporar nuevos conocimientos y establecer consensos de nomenclatura y métodos de registro para el reconocimiento en restos humanos arqueológicos, a diferencia de lo que ocurre en poblaciones actuales, en las cuales el diagnostico resulta más preciso y consensuado (e.g. Aichroth 1971; Anderson 2001; Cahill 1995; Edmonds y Polousky 2013; Takahara et al. 1999). En este sentido, resulta importante la inclusión de información proveniente de estudios clínicos con muestras de pacientes hospitalarios y de estudios veterinarios, la cual puede ofrecer abundante conocimiento acerca de la etiología, patogénesis y consecuencias de la OCD (e.g. Edmonds y Heyworth 2014; Schenck y Goodnight 1996 Ytrehus et al. 2007) y de esa manera contribuir a mejorar los diagnósticos diferenciales realizados a partir del análisis de restos osteológicos.

Sumado a estos problemas, ha habido una escasa evaluación de la OCD en conjuntos esqueletales arqueológicos en Argentina, provenientes de poblaciones antiguas. El trabajo presentado aquí busca hacer un aporte inicial en este sentido, en una de las regiones en las cuales se describieron lesiones por OCD anteriormente, como Patagonia Austral. La prevalencia de OCD observada en este estudio es mayor a la identificada en otros conjuntos de restos humanos provenientes tanto de contextos arqueológicos como de estudios clínicos (Tabla 1). Las prevalencias más próximas a las encontradas aquí son las reportadas en estudios sobre atletas, en los cuales la OCD está vinculada con la actividad física. Por lo tanto, es posible sugerir que la alta frecuencia identificada en Patagonia Austral podría estar asociada con este aspecto más que con otras causas, como factores hereditarios o la predisposición morfológica de las articulaciones. Sin embargo, no puede descartarse que esta elevada frecuencia pueda también incluir sesgos asociados a características de la muestra como la distribución sexual y etaria.

Por otra parte, las articulaciones más afectadas en

Tabla 4. Cantidad de individuos con lesiones y prevalencias de OCD en el conjunto de restos humanos provenientes de Patagonia Austral

Table 4. Number of individuals with lesions and prevalences of $O C D$ in the sample of human remains from Southern Patagonia

\begin{tabular}{lccc}
\hline & N afectado & N observado & $\%$ \\
\hline $\begin{array}{l}\text { Total } \\
\text { Sexo }\end{array}$ & 11 & 26 & 42,3 \\
$\quad$ Masculino & 10 & 19 & 52,6 \\
$\quad$ Femenino & 1 & 7 & 14,3 \\
Edad & & & \\
$\quad$ Adulto joven (20-35) & 7 & 19 & 36,8 \\
$\quad$ Adulto medio (35-50) & 4 & 7 & 57,1 \\
Cronología & & & \\
$\quad$ Pre-contacto & 5 & 11 & 45,4 \\
$\quad$ Durante el contacto & 5 & 12 & 41,7 \\
Dieta & & & \\
$\quad$ Terrestre & 8 & 13 & 61,5 \\
$\quad$ Mixta & 2 & 5 & 40 \\
$\quad$ Marítima & 1 & 5 & 20 \\
Región & & & \\
$\quad$ Santa Cruz/Magallanes & 6 & 10 & 60 \\
$\quad$ Norte de Tierra del Fuego & 4 & 9 & 44,4 \\
$\quad$ Sur de Tierra del Fuego & 1 & 7 & 14,3 \\
\hline
\end{tabular}


el conjunto analizado aquí no se corresponden con aquellas en las que la OCD ha sido más diagnosticada en poblaciones actuales. No fueron identificadas lesiones compatibles con OCD en las facetas articulares que componen la rodilla y el tobillo, mientras que en solo un individuo (Rincón del Buque; Tabla 3) se registró una lesión en el codo. Por el contrario, en este conjunto se destacan las OCD asociadas con la articulación del hombro, que afectaron a 8 individuos, 2 de los cuales presentaron esta lesión de manera bilateral.

Por otra parte, la OCD en la falange proximal del pie fue registrada en dos individuos, en uno de ellos de forma bilateral. La OCD en esta articulación, a pesar de ser muy poco frecuente en los estudios clínicos, ha sido observada en diferentes contextos bioarqueológicos (McWhirr et al. 1982; Rodríguez-Martín 2000; Vikatou et al. 2017; Wells 1974), con prevalencias que alcanzan hasta un 17,9\% (McWhirr et al. 1982). De acuerdo con Wells (1974), su presencia podría deberse a microtraumatismos causados por actividades que involucran movimientos de carga, empuje y giro, y el uso recurrente de calzados que impiden una flexión natural del pie, aunque no debe descartarse por completo la influencia de factores genéticos o hereditarios (Vikatou et al. 2017; Wells 1974). En este estudio las lesiones en esta articulación se presentaron en dos individuos del periodo de contacto, ambos asociados con el consumo de recursos terrestres (Juni Aike y Lengua de Vaca). Por lo tanto, sería posible sugerir que las actividades físicas involucradas en este tipo de economía durante este periodo podrían tener alguna influencia en el desarrollo de OCD en esta articulación, aunque el reducido número de individuos estudiados requiere nuevas investigaciones que incrementen la muestra analizada para poner a prueba esta hipótesis.

Por el contrario, la OCD en el codo, como es el caso de la epífisis proximal del cúbito del individuo procedente de Rincón del Buque (Tabla 3), ha sido poco documentada en estudios bioarqueológicos previos (McWhirr et al. 1982; Rodríguez-Martín 2000; Silva 2001; Wells 1974). En cambio, fue ampliamente tratada por la literatura clínica del comportamiento corporal de los deportistas (e.g. Kida et al. 2014; Matsuura et al. 2014) debido a que las lesiones en esta articulación suelen estar asociadas con el movimiento de lanzamiento (Guerra y Timmerman 1996). En este caso se trata de un individuo masculino, con dieta predominantemente terrestre. Por lo tanto, al igual que en los casos descriptos más arriba, podría especularse que la lesión observada en este individuo podría estar relacionada con algún tipo de actividad involucrada como parte de la economía terrestre y que implicara una mayor carga mecánica sobre la articulación del codo.

Finalmente, la presencia de OCD en vértebras, como en el axis del individuo del sitio Juni Aike (Tabla 3), ha sido documentada solo en pocas ocasiones en poblaciones antiguas (McWirr et al. 1982; Wells 1974). Esta podría estar relacionada con microtraumatismos repetitivos, aunque dada su escasa frecuencia en poblaciones actuales se desconoce el tipo de actividad que pudiera causar ese daño en la articulación, por lo que resulta aventurado por el momento asociarla a algún patrón de actividad o estrategia económica en este individuo en particular. Este es el único individuo en el cual se registró OCD en más de una articulación diferente, dado que también presentó una lesión bilateral de este tipo en la primera falange del primer dedo.

A pesar de las diferencias observadas en las prevalencias asociadas al sexo, a la edad, a la distribución geográfica y cronológica y a la paleodieta, en ningún caso estas diferencias fueron estadísticamente significativas. Los individuos de sexo masculino presentaron un mayor porcentaje de OCD que los femeninos, lo que se condice con los antecedentes clínicos (Kocher et al. 2006; Schenck y Goodnight 1996) y arqueológicos (Aufderheide y Rodríguez-Martín 1998; Ortner, 2003; Waldron 2009). No es posible descartar que este resultado se encuentre sesgado por la baja cantidad de individuos femeninos existentes en la muestra analizada, por lo que estudios con muestras más numerosas y homogéneas entre sexos permitirán discutir en mayor detalle la distribución sexual de la OCD en Patagonia Austral.

Respecto a la edad, los individuos adultos medios presentaron una mayor frecuencia de OCD respecto de los adultos jóvenes, lo cual se contrapone con la tendencia observada en estudios arqueológicos previos (Aufderheide y Rodríguez-Martin 1998; Ortner 2003; Rogers y Waldron 1995) y clínicos (Aichroth 1971; Takahara et al. 1999). Sin embargo, no es posible descartar que al menos algunas de las OCD identificadas hayan sido producidas durante la adolescencia o principios de la adultez de los individuos, periodo en el cual se ha documentado que existe una mayor susceptibilidad de aparición (Cahill 1995; Edge y Porter 2011; Garrett 1991; Vikatou et al. 2017).

Respecto a la distribución regional, se observó una mayor prevalencia de OCD en el área de Santa Cruz/Magallanes y el Norte de Tierra del Fuego que en el Sur de Tierra del Fuego. Además, los individuos con dietas terrestres presentaron las mayores prevalencias, seguidos por aquellos con dietas mixtas, y en menor medida marítimas. En las regiones correspondientes al Norte, principalmente representadas por individuos con dietas terrestres y mixtas, la articulación más frecuentemente afectada es la fosa glenoidea del omóplato. De acuerdo con estudios previos (Bullough 2004; Edmonds y Heyworth 2014; Edmonds y Polousky 2013; Waldron 2009), esta articulación no es frecuentemente afectada por la OCD en poblaciones tanto actuales como pasadas, aunque se ha reportado en unos pocos individuos a partir de estudios bioarqueológicos (Bourbou 2003; McWirr et al. 1982; Rodríguez-Martín 2000) y clínicos (Chu et al. 2009; Gogus y Ozturk 2008; Shanley y Mulligan 1990). 
Por el contrario, estudios realizados en individuos que desarrollan deportes que involucran el uso intenso del hombro muestran mayores prevalencias de OCD en esa articulación (Edmonds y Heyworth 2014). Por lo tanto, es posible plantear que estas lesiones serían consecuencia de actividades que formaron parte de una economía de subsistencia terrestre. Los estudios etnográficos (e.g. Gusinde 1986) mencionan extensamente el uso del arco y la flecha por parte de los hombres, de manera que la hipótesis de que la alta frecuencia de OCD en el omóplato se debe a algún tipo de actividad física que involucra el uso de ambos miembros superiores, como la arquería implementada durante la caza por las poblaciones con economías terrestres y mixtas en Patagonia Austral, parece plausible. Aunque en este trabajo estas lesiones afectaron solo a individuos masculinos, este resultado podría estar sesgado por el reducido número de esqueletos de sexo femenino, por lo que es necesario contrastarlo con conjuntos de mayor tamaño. Respecto a los individuos recuperados en los sitios Caleta Falsa 8-1 y Bahía Felipe 1, dado a que la OCD del hombro se presentó de manera bilateral, podría estar asociada además con procesos de isquemia del cartílago articular durante el crecimiento o la vejez, tal como fue sugerido por Carlson et al. (1991) e Ytrehus et al. (2004) para estos casos.

La mayor frecuencia de OCD observada en individuos del norte de Tierra del Fuego y el área de Santa Cruz/Magallanes, preferentemente caracterizada por dietas terrestres y mixtas, parece acompañar algunos resultados preliminares previos en los cuales se observó una mayor prevalencia de lesiones articulares tales como osteoartrosis en los miembros superiores (Suby 2014a), nódulos de Schmorl sobre la columna vertebral dorsolumbar (Suby 2014b) y espondilólisis (D'angelo del Campo et al. 2017) en muestras de individuos con estas economías respecto a aquellos con economías asociadas a recursos marítimos. Por lo tanto, los resultados obtenidos aquí acompañarían la hipótesis de una mayor actividad física en las poblaciones con estrategias de subsistencia asociadas a actividades terrestres que deriven en problemas articulares como la osteoartrosis, los nódulos de Schmorl y la osteocondritis disecante.

Por otro lado, la menor frecuencia de OCD en la muestra del Sur de Tierra del Fuego, que contiene una mayor cantidad de individuos asociados con dietas marítimas, indica que las actividades vinculadas con este tipo de economía no habrían contribuido al desarrollo de OCD en las articulaciones del hombro, del codo y del pie. Según información etnográfica (e.g. Gusinde 1986; Orquera y Piana 1999; Saletta 2015), las mujeres Ilevaban a cabo actividades de remo en forma más frecuente que los hombres. Al igual que en el Norte, en este estudio solo fueron analizados restos de tres mujeres pertenecientes al archipiélago Sur de Tierra del Fuego. Por lo tanto, no es posible descartar sesgos muestrales que conduzcan a la baja prevalencia de OCD en el hombro para los individuos de esta área.
En relación con la cronología, no se observaron diferencias en las prevalencias de OCD al comparar los individuos pertenecientes a momentos previos y los que vivieron durante el contacto. Estos primeros resultados parecen señalar que, de haber existido cambios en el comportamiento de los individuos con cronologías posteriores al contacto (e.g. Borrero 2001; Guichón et al. 2006), no habrían redundado en un incremento en la prevalencia de OCD. No es posible por el momento establecer qué ocurrió en otros contextos históricos, como en el caso de las personas que habitaron las misiones religiosas, las cuales habrían modificaron sus actividades físicas y económicas (e.g. Casali 2011; García Laborde et al. 2010). Estos cambios podrían haber resultado en modificaciones de la prevalencia de OCD respecto de las presentadas en este trabajo para el periodo posterior al contacto.

\section{Conclusiones}

Las causas asociadas a las OCD son múltiples y en la mayoría de las circunstancias resulta difícil discriminar entre ellas. La prevalencia general observada en esta muestra, mayor a la reportada para poblaciones actuales, sugiere que la actividad física habría desempeñado un rol importante en el desarrollo de la OCD en al menos parte de los individuos afectados, en especial en aquellos en los cuales fueron afectadas articulaciones como las del hombro y el codo. Esta hipótesis podría ser sustentada a su vez por los reportes recientes que señalan que las poblaciones de Santa Cruz/Magallanes y el Norte de Tierra del Fuego habrían estado afectadas por mayores acciones mecánicas sobre la columna vertebral que en las poblaciones del Sur de Tierra del Fuego, posiblemente asociadas a la actividad física ( $D$ 'angelo del Campo et al. 2017; Suby 2014b). De todas formas, no se descartan otros posibles factores como componentes genéticos hereditarios (Padgett et al. 1995), variaciones anatómicas en las articulaciones (Bulgheroni et al. 2017) o isquemia de los cartílagos articulares durante el período de crecimiento y vejez de los individuos (Carlson et al. 1991; Ytrehus et al. 2004).

El estudio de la OCD ha sido ampliamente desarrollado en la literatura clínica y veterinaria. Como resultado, se ha generado un abundante conocimiento que puede ser utilizado en el estudio de restos bioarqueológicos. Las dificultades planteadas respecto de la terminología, la metodología de análisis y el diagnóstico implican desafíos particulares, que en el futuro será necesario ampliar para proponer caminos apropiados para su análisis e interpretación. Al mismo tiempo, será de utilidad para discutir diferentes aspectos relacionados con la actividad física de las poblaciones humanas del pasado. Este trabajo involucra un primer paso en el estudio de esta patología en restos humanos de Patagonia Austral, la cual, a pesar de algunas menciones descriptivas previas aisladas, no fue analizada en forma sistemática. Dado el reducido número de restos humanos incluidos en el análisis, en el 
futuro serán necesarios nuevos estudios que incluyan un mayor conjunto de individuos.

Buenos Aires, Necochea y Olavarría, 15 de diciembre de 2017

\section{Agradecimientos}

Agradecemos a los Dres. Luis Borrero, Ricardo Guichón, Mónica Salemme, Fernando Santiago, Ernesto Piana y Francisco Zangrando por el acceso a los restos recuperados en el marco de sus respectivos proyectos de investigación. Al Instituto de la Patagonia (Universidad de Magallanes, Chile), por facilitar el acceso a los restos resguardados en esa institución. A los dos revisores anónimos que con sus comentarios contribuyeron a mejorar la calidad del trabajo. Este trabajo fue financiado por los proyectos PICT 2008-0385 y 2016-0191.

\section{Referencias}

Aichroth, P. 1971. Osteochondritis dissecans of the knee. Bone \& Joint Journal 53(3): 440-447.

Anderson, T. 2000. A medieval Italian child with osteochondritis dissecans of the cuboid. The Foot 10(4): 216-218.

Anderson, T, 2001. An example of unhealed osteochondritis dissecans of the medial cuneiform. International Journal of Osteoarchaeology 11: 381-384.

Appleby, J., R. Thomas y J. Buikstra. 2015. Increasing confidence in paleopathological diagnosis. Application of the Istanbul terminological framework. International Journal of Paleopathology 8: 19-21.

Aufderheide, A. y C. Rodríguez-Martín. 1998. The Cambridge encyclopedia of human paleopathology. Cambridge University Press, Cambridge, Reino Unido.

Barberena, R. 2002. Los límites del mar. Sociedad Argentina de Antropología, Buenos Aires.

Barrie, H. J. 1987. Osteochondritis dissecans 1887-1987. A centennial look at König's memorable phrase. Bone $\&$ Joint Journal 69(5): 693-695.

Berndt, A. y M. Harty. 1959. Transchondral fractures (Osteochondritis Dissecans) of the talus. The Journal of Bone and Joint Surgery. American Volume 41: 988-1020.

Borrero, L. 2001. El poblamiento de la Patagonia. Toldos, milodones y volcanes. Emecé, Buenos Aires.

Borrero, L. y R. Barberena. 2006. Hunter-gatherer home ranges and marine resources: An archaeological case from southern Patagonia. Current Anthropology 47(5): 855-867.
Borrero, L., R. Barberena, N. Franco, J. Charlin y R. Tykot. 2009. Isotopes and rocks: geographical organization of Southern Patagonian hunter-gatherers. International Journal of Osteoarchaeology 19: 309-327.

Bourbou, C. 2003. Health patterns of proto-Byzantine populations (6th-7th centuries AD) in south Greece: the cases of Eleutherna (Crete) and Messene (Peloponnese). International Journal of Osteoarchaeology 13(5): 303313.

Brooks, S. y J. Suchey. 1990. Skeletal age determination based on the os pubis: a comparison of the AcsádiNemeskéri and Suchey-Brooks methods. Human Evolution 5(3): 227-238.

Brownlow, H., L. O'Connor-Read y M. Perko. 2006. Arthroscopic treatment of osteochondritis dissecans of the capitellum. Knee Surgery, Sports Traumatology, Arthroscopy 14(2): 198-202.

Bruzek, J. 2002. A method for visual determination of sex, using the human hip bone. American Journal of Physical Anthropology 117: 157-168.

Buikstra, J. y D. Ubelaker. 1994. Standards for data collection from human skeletal remains. Arkansas Archaeological Survey Research Series N44. Arkansas, USA.

Bulgheroni, E., L. Mattioli y P. Bulgheroni. 2017. Evolution of Osteochondritis Dissecans of the lateral femoral condyle combined with discoid meniscus. Joints 5(2): 114-117.

Bullough, P. 2004. Orthopaedic pathology. Elsevier Health Sciences, Missouri, USA.

Cahill, B. 1995. Osteochondritis dissecans of the knee: treatment of juvenile and adult forms. Journal of the American Academy of Orthopaedic Surgeons 3(4): 237247.

Capasso, L., K. Kennedy y C. Wilczak. 1998. Atlas of occupational markers on human remains. Journal of Paleontology. Monographic Publication 3. Edigrafital SPA, Terramo, Italia.

Carlson, C., D. Meuten y D. Richardson. 1991. Ischemic necrosis of cartilage in spontaneous and experimental lesions of osteochondrosis. Journal of Orthopaedic Research 9(3): 317-329.

Casali, R. 2011. Contacto interétnico en el Norte de Tierra del Fuego: la Misión Salesiana La Candelaria (Río Grande) y la salud de la población Selk'nam (1895-1931). Facultad de Humanidades, Universidad Nacional de Mar del Plata, Argentina. Tesis Doctoral inédita, Buenos Aires. 
Chu, P., J. Shih, Y. Hou, S. Hung, J. Chen y H. Lee. 2009. Osteochondritis dissecans of the glenoid: a rare injury secondary to repetitive microtrauma. Journal of Trauma and Acute Care Surgery 67(3): E62-E64.

D’angelo del Campo, M., J. Suby, P. García-Laborde y R. Guichón. 2017. Spondylolysis in the past: a case study of hunter-gatherers from Southern Patagonia. International Journal of Paleopathology 19: 1-17.

Darton, Y., V. Gallien, É. Henrion, M. Dupuis e I. Richard 2017. Osteochondral lesions and partial fractures of the talus in paleopathology. International Journal of Paleopathology 16: 31-39.

Debeer, P. y P. Brys. 2005. Osteochondritis dissecans of the humeral head: clinical and radiological findings. Acta Orthopaedica Belgica 71(4): 484-488.

Desai, S., M. Patel, L. Michelli, J. Silver y R. Lidge. 1987. Osteochondritis dissecans of the patella. Bone \& Joint Journal 69(2): 320-325.

Edge, A. y K. Porter. 2011. Osteochondritis dissecans: a review. Trauma 13(1): 23-33.

Edmonds, E. y B. Heyworth. 2014. Osteochondritis dissecans of the shoulder and hip. Clinics in Sports Medicine 33(2): 285-294.

Edmonds, E. y J. Polousky. 2013. A review of knowledge in osteochondritis dissecans: 123 years of minimal evolution from König to the ROCK study group. Clinical Orthopaedics and Related Research Research 471: 1118-1126.

Eygendaal, D., G. Bain, L. Pederzini y G. Poehling. 2017. Osteochondritis dissecans of the elbow: state of the art. Journal of ISAKOS: Joint Disorders \& Orthopaedic Sports Medicine 2(1): 47-57.

Flensborg, G., G. Martínez, M. González y P. Bayala. 2011. Revisión de los restos óseos humanos del sitio La Petrona (Transición Pampeano-Patagónica oriental, Argentina). Magallania 39(1): 179-191.

García Laborde P., J. Suby, R. Guichón y R. Casali. 2010. El antiguo cementerio de la misión de Rio Grande, Tierra del Fuego. Primeros resultados sobre patologías nutricionales-metabólicas e infecciosas. Revista Argentina de Antropología Biológica 12: 57-69.

Garrett, J. 1991. Osteochondritis dissecans. Clinics in Sports Medicine 10(3): 569-593.

Gogus, A. y C. Ozturk. 2008. Osteochondritis dissecans of the glenoid cavity: a case report. Archives of Orthopaedic and Trauma Surgery 128(5): 457-460.
González-José, R., S. Dahinten, M. Hernández y H. Pucciarelli. 2001. Craneometric variation and the settlement of the Americas: testing hypotheses by means of R-matrix and matrix correlation analyses. American Journal of Physical Anthropology 116: 154-165.

Guerra, J. y L. Timmerman. 1996. Clinical anatomy, histology and pathomechanics of the elbow in sports. Operative Techniques in Sports Medicine 4(2): 69-76.

Guichón, R. 1994. “Antropología física de Tierra del Fuego, caracterización biológica de las poblaciones prehispánicas". Facultad de Filosofía y Letras, Universidad de Buenos Aires, Argentina. Tesis Doctoral inédita, Buenos Aires.

Guichón, R., A. Muñoz y L. Borrero. 2000. Datos para una tafonomía de restos óseos humanos en Bahía San Sebastián, Tierra del Fuego. Relaciones de la Sociedad Argentina de Antropología XXV: 297-311.

Guichón, R. y J. Suby. 2011. Estudio bioarqueológico de los restos humanos recuperados por Anne Chapman en Caleta Falsa, Tierra del Fuego. Magallania 39(1): 163-177.

Guichón, R., J. Suby, R. Casali y M. Fugassa. 2006. Health at the time of native-European contact in Southern Patagonia. Memorias do Instituto Oswaldo Cruz 101(Suppl. II): 97-105.

Gusinde, M. 1986. Los indios de Tierra del Fuego. Los Yamana. Centro Argentino de Etnología Americana, Buenos Aires.

Hefti, F., J. Beguiristain, R. Krauspe, B. Moller-Madsen, V. Riccio, C. Tschauner, R. Wetzel y R. Zeller. 1999. Osteochondritis dissecans: a multicenter study of the European Pediatric Orthopedic Society. Journal of Pediatric Orthopaedics B 8: 231-245.

Hendrix, C. 2005. Calcaneal apophysitis (Sever disease). Clinics in Pediatric Medicine and Surgery 22(1): 55-62.

Jaberi, F. 2002. Osteochondritis dissecans of the weightbearing surface of the medial femoral condyle in adults. The Knee 9(3): 201-207.

Kessler, J., H. Nikizad, K. Shea, J. Jacobs y J. Weiss. 2013. The demographics and epidemiology of osteochondritis dissecans of the ankle, elbow, foot, and shoulder in children. Orthopaedic Journal of Sports Medicine 1(4 suppl.): 2325967113500024.

Kida, Y., T. Morihara, Y. Kotoura, T. Hojo, H. Tachiiri, T. Sukenari, Y. Iwata, R. Furukawa, R. Oda, Y. Arai, H. Fujiwara y T. Kubo. 2014. Prevalence and clinical characteristics of osteochondritis dissecans of the humeral capitellum among adolescent baseball players. The 
American Journal of Sports Medicine 42(8): 1963-1971.

Kocher, M., R. Tucker, T. Ganley y J. Flynn. 2006. Management of osteochondritis dissecans of the knee. The American Journal of Sports Medicine 34(7): 11811191.

König, F. 2013. The classic: on loose bodies in the joint. Clinical Orthopaedics and Related Research 471(4): 1107-1115.

Kothari, A., P. Ponce, B. Arriaza y L. O'Connor-read. 2009. Osteochondritis dissecans of the knee in a mummy from Northern Chile. The Knee 16: 159-160.

Krogman, W. y M. Iscan. 1986. The human skeleton in forensic science. CC Thomas, Springfield.

L'Heureux, L. y R. Barberena. 2008. Evidencias bioarqueológicas en Patagonia Meridional: el sitio Orejas de Burro 1 (Pali Aike, provincia de Santa Cruz). Intersecciones en Antropología 9: 11-24.

L'Heureux, L., R. Guichón, R. Barberena y L. Borrero. 2003. Durmiendo bajo el faro: estudio de un entierro humano en Cabo Vírgenes (CV 17), provincia de Santa Cruz, República Argentina. Intersecciones en Antropología 4: 87-97.

Lindén, B. 1976. The incidence of osteochondritis dissecans in the condyles of the femur. Acta Orthopaedica Scandinava 47: 664-667.

Lovejoy, C., R. Meindl, R. Mensforth y T. Barton. 1985. Multifactorial determination of skeletal age at death: a method and blind tests of its accuracy. American Journal of Physical Anthropology 68: 1-14.

Loveland, C., J. Gregg y W. Bass. 1984. Osteochondritis dissecans from the Great Plains of North America. The Plains Anthropologist 29: 239-246.

Lowe, T. 1990. Scheuermann disease. The Journal of Bone and Joint Surgery. American 72(6): 940-945.

Matsuura, T., N. Suzue, T. Iwame, S. Nishio y K. Sairyo, K. 2014. Prevalence of Osteochondritis Dissecans of the capitellum in young baseball players: results based on ultrasonographic findings. The Orthopaedic Journal of Sports Medicine 2(8): 1-5.

McWhirr, A., L. Viner y C. Wells. 1982. Romano-British cemeteries at Cirencester (Vol. 2). Cirencester Excavation Committee Corinium Museum, Greece.

Meindl, R. y C. Lovejoy. 1989. Age changes in the pelvis: implications for paleodemography. Isçan, M. (ed.), Age markers in the human skeleton, 137-168, Ch. C. Thomas Publisher. Springfield, Illinois.
Ming, S., D. Jin y M. Kanta. 2004. Arthroscopic treatment of osteochondritis dissecans of the talus. Foot and Ankle Surgery 10(4): 181-186.

Nagura, S. 1960. The so-called osteochondritis dissecans of König. Clinical Orthopaedics and Related Research 18: $100-122$.

O'Connor, T. 2008. On the differential diagnosis of arthropathy in bovids. Grupe, G., G. McGlynn y J. Peters (eds.), Limping together through the ages. Joint afflictions and bone infections, Documenta Archaeobiologae 6, 165-186, Verlag Marie Leidorf, Rahden.

Orquera, L. y E. Piana. 1999. Sea nomads of the Beagle Channel in Southernmost South America: over six thousand years of coastal adaptation and stability. The Journal of Island and Coastal Archaeology 4(1): 61-81.

Ortner, D. 2003. Identification of pathological conditions in human skeletal remains. Segunda edición. Elsevier Science/Academic Press, Nueva York.

Osborne, D., L. Simmons y S. Nawrocki. 2004. Reconsidering the auricular surface as an indicator of age at death. Journal of Forensic Sciences 49(5): 1-7.

Padgett, G., U. Mostosky, C. Probst, M. Thomas y C. Krecke. 1995. The inheritance of osteochondritis dissecans and fragmented coronoid process of the elbow joint in labrador retrievers. Journal of the American Animal Hospital Association 31(4): 327-330.

Paget, J. 1870. On the production of some of the loose bodies in joints. St. Bartholomews Hospital Reports 6: $1-4$.

Petersen, J., J. Steinhagen, P. Catala-Lehnen y J. Bruns. 2006. Osteochondritis dissecans of the knee joint. Zeitschrift fur Orthopadie und ihre Grenzgebiete 144(4): R63-76.

Piana, E., A. Tessone y A. Zangrando. 2006. Contextos mortuorios en la región del canal Beagle... del hallazgo fortuito a la búsqueda sistemática. Magallania 1: 87-101.

Polousky, J. 2011. Juvenile Osteochondritis Dissecans. Sports Medicine and Arthroscopy Review 19(1): 56-63.

Ponce, P. 2010. "A comparative study of activity-related skeletal changes in 3rd-2nd millennium BC coastal fishers and 1st millenium AD inland agriculturists in Chile, South America". Tesis Doctoral inédita, Durham University.

Prieto, A. 1993-1994. Algunos datos en torno a los enterratorios humanos de la región continental de Magallanes. Anales del Instituto de la Patagonia 22: 91-100. 
Ratto, N., C. Aranda y L. Luna 2018. Caracterización inicial de las prácticas mortuorias, osteobiografía y síndrome criboso en Las Papas (Fiambalá, Catamarca, Argentina). Actas del XX Congreso Nacional de Arqueología Chilena. Concepción. Chile. En prensa.

Rodríguez-Martín, C. 2000. Osteocondritis disecante en poblaciones del pasado. Una revisión sobre su etiología, fisiopatología y epidemiología, con especial referencia a Canarias. Eres (Serie de Arqueología-Bioantropología) 9: 201-219.

Rogers, J. y T. Waldron. 1995. A field guide to joint disease in archaeology. John Wiley \& Sons, Nueva York.

Saletta, M. J. 2015. “Excavando fuentes. La tecnología, subsistencia, movilidad y los sistemas simbólicos de Shelk'nam. Yámana/Yaghan y Aonikenk entre los siglos XVI y XX analizadas a partir de los registros escritos y arqueológicos". Facultad de Filosofía y Letras, Universidad de Buenos Aires, Argentina. Tesis Doctoral inédita, Buenos Aires.

Solomon, L., D. Warwick y S. Nayagam (eds.). 2010. Apley's system of orthopaedics and fractures. CRC Press, Nueva York.

Santiago, F., M. Salemme, J. Suby y R. Guichón. 2011. Restos humanos en el Norte de Tierra del Fuego: aspectos contextuales, dietarios y paleopatológicos. Intersecciones en Antropología 12(1): 147-162.

Scabuzzo, C. 2010. "Actividad, patología y nutrición de los cazadores-recolectores pampeanos". Facultad de Ciencias Naturales y Museo, Universidad Nacional de La Plata, Tesis Doctoral inédita, La Plata.

Schenck, R. y J. Goodnight. 1996. Osteochondritis dissecans. Journal of Bone and Joint Surgery 78: 439-456.

Schimmer, R., W. Dick y B. Hintermann. 2001. The role of ankle arthroscopy in the treatment strategies of osteochondritis dissecans lesions of the talus. Foot $\&$ Ankle International 22(11): 895-900.

Shanley, D. y M. Mulligan. 1990. Osteochondrosis dissecans of the glenoid. Skeletal Radiology 19(6): 419421.

Silva, A. M. 2001. Evidence of osteochondritis dissecans in late Neolithic/Chalcolithic Portuguese populations. Actas del VI Congreso Nacional de Paleopatología: 464-468. Asociación Española de Paleopatología, Madrid.

Slaus, M., Cicvara Pećina, I. Lucijanić y D. Strinović. 2010. Osteochondritis Dissecans of the knee in a subadult from a Medieval (Ninth Century AD) site in Croatia. Acta Clinica Croatica 49(2): 189-196.
Stirland, A. 1996. Patterns of trauma in a unique medieval parish cemetery. International Journal of Osteoarchaeology 6(1): 92-100.

Suby, J. 2014a. Desarrollos recientes en el estudio de la salud de las poblaciones humanas antiguas de Patagonia Austral. Luna, L., C. Aranda y J. Suby (eds.), Avances recientes en la bioarqueología latinoamericana, 69-100. Grupo de Investigación en Bioarqueología, Buenos Aires.

Suby, J. 2014b. Nódulos de Schmorl en restos humanos arqueológicos de Patagonia Austral. Magallania 42(1): 135-147.

Suby, J., R. Guichón y A. Zangrando. 2009. El registro biológico humano de la costa meridional de Santa Cruz. Revista Argentina de Antropología Biológica 11(1): 109-124.

Suby J., A. Zangrando y E. Piana. 2011. Exploraciones osteológicas de la salud de las poblaciones humanas del Canal Beagle. Relaciones de la Sociedad Argentina de Antropología 36: 249-270.

Suchey, J. y D. Katz. 1998. Applications of pubic age determination in a forensic setting. Reichs K. (ed.), Forensic osteology: advances in the identification of human remains, 204-236. Charles C. Thomas, Springfield, Illinois.

Takahara, M., T. Ogino, I. Sasaki, H. Kato, A. Minami y K. Kaneda. 1999. Long term outcome of osteochondritis dissecans of the humeral capitellum. Clinical Orthopaedics and Related Research 363: 108-115.

Tessone, A., R. Guichón, J. Suby y L. Kozameh. 2011. Bioarqueología de Península Mitre. Zangrando, A., M. Vázquez y A. Tessone (eds.), Los cazadores-recolectores del extremo oriental fueguino. Arqueología de Península Mitre e Isla de los Estados, 231-270. Sociedad Argentina de Antropología, Buenos Aires.

Thomas, R. y N. Johannsen. 2011. Articular depressions in domestic cattle phalanges and their archaeological relevance. International Journal of Paleopathology 1(1): 43-54.

Todd, T. 1921. Age changes in the pubic bone. American Journal of Physical Anthropology 4(1): 1-70.

Uematsu, K., T. Habata, Y. Hasegawa, K. Hattori, R. Kasanami, Y. Takakura y Y. Fujisawa. 2005. Osteochondritis dissecans of the knee: long-term results of excision of the osteochondral fragment. The Knee 12(3): 205-208.

Vikatou, I. 2012. "Are these 'clogs' made for walking? Osteochondritis dissecans: evidence of strenuous activity and trauma on skeletal elements of the foot from a post- 
medieval rural society in the Netherlands". Facultad de Arqueología, Universidad de Leiden, Tesis de Maestría inédita. Holanda.

Vikatou, I., M. Hoogland y A. Waters-Rist. 2017. Osteochondritis Dissecans of skeletal elements of the foot in a 19th century rural farming community from The Netherlands. International Journal of Paleopathology 19: 53-63.

Waldron, T. 2009. Paleopathology. Cambridge University Press, Cambridge.

Wells, C. 1962. Joint pathology in ancient Anglo-Saxons.
Bone \& Joint Journal 44(4): 948-949.

Wells, C. 1974. Osteochondritis dissecans in ancient British skeletal material. Medical History 18: 365-369.

Ytrehus, B., C. Carlson y S. Ekman. 2007. Etiology and pathogenesis of osteochondrosis. Veterinary Pathology 44(4): 429-448.

Ytrehus, B., C. Carlson, N. Lundeheim, L. Mathisen, F. Reinholt, J. Teige y S. Ekman. 2004. Vascularisation and osteochondrosis of the epiphyseal growth cartilage of the distal femur in pigs-development with age, growth rate, weight and joint shape. Bone 34(3): 454-465. 